\title{
PROCESS MAPPING OF LASER SURFACE MODIFICATION OF AISI 316L STAINLESS STEEL FOR BIOMEDICAL APPLICATIONS
}

\author{
Evans Chikarakara, Sumsun Naher, Dermot Brabazon \\ Materials Processing Research Centre, Dublin City University, Dublin 9, Ireland \\ e-mail: evans.chikarakara2@,mail.dcu.ie
}

\begin{abstract}
A $1.5-\mathrm{kW} \mathrm{CO}_{2}$ laser in pulsed mode at $3 \mathrm{kHz}$ was used to investigate the effects of varied laser process parameters and resulting morphology of AISI $316 \mathrm{~L}$ stainless steel. Irradiance and residence time were varied between 7.9 to $23.6 \mathrm{MW} / \mathrm{cm}^{2}$ and 50 to $167 \mu$ s respectively. A strong correlation between irradiance, residence time, depth of processing and roughness of processed steel was established. The high depth of altered microstructure and increased roughness were linked to higher levels of both irradiance and residence times. Energy fluence and surface temperature models were used to predict levels of melting occurring on the surface through the analysis of roughness and depth of the region processed. Microstructural images captured by the SEM revealed significant grain structure changes at higher irradiances, but due to increased residence times, limited to the laser in use, the hardness values were not improved.
\end{abstract}

PACS: 87.85.J, 68.37.Hk, 81.65.-b

\section{INTRODUCTION}

The main metal groups dominating in biomedical implants are 316L stainless steel, cobalt-chrome-molybdenum alloys, pure titanium and titanium alloys, due to their strength, corrosion resistance, biocompatibility and reasonable cost. The corrosion and wear resistance of metallic implants is fairly high albeit that the creation of wear debris is an ongoing concern in relation to life of total joint replacements. [1,2]. Due to limited lifetime for biomedical implants, material engineers have strived to improve the surface properties of existing biomaterials. Internal stresses make it difficult to bond CVD, PVD and DLC coatings to the substrates, thus weakening the structure and limiting the life of implants [3]. Scanning high-power density lasers on a workpiece can cause the heated surface to melt, while varying the exposure time dictates heating and cooling regimes therefore controlling the resulting surface microstructures. The surface can thus be treated for glazing, alloying, cladding, improving biocompatibility and material homogenization $[4,5]$. Advantages presented by laser surface treatment include: superior bonding, reduced distortion, improved physical properties (hardness levels, wear and corrosion resistance due to rapid quenching), simple oxidation elimination techniques and easier control over depth of processing [6]. Conventional processing of $316 \mathrm{~L}$ can provide crystalline anisotropy and inter-crystalline defects. The latter crystalline features can provide the dislocation nucleation sites and shorter diffusion paths for enhanced oxidation and corrosion degradation [7, 8]. Laser treatments can provide more homogenous structures and amorphisation which can overcome this latter problem. Various authors have achieved improved hardness, wear and corrosion rates on the surface of metal through laser irradiation [9-11].

Surface roughness of biomedical implants has a significant influence on the tissue reaction at the interface. The average surface mean roughness used in biomedical implants for bone fixation is between $1.5-4 \mu \mathrm{m}$, however, the value varies depending on the application $[12,13]$. Some studies have indicated that rougher surfaces have stimulated higher bone resorption as compared to smoother surfaces [14]. Bone resorption generally results in loosening of implants. In addition, increased depth of surface modification is advantageous since the resulting surface can be ground to preferred roughness levels.

The current study investigates laser processing with parameters within the laser glazing region on 316L stainless steel. Laser processing parameters effects on surface temperature, microstructure, melt pool size and roughness were investigated.

\section{EXPERIMENTAL}

AISI 316L austenitic stainless steel, with elemental composition given in Table 1, was surface treated using a 1.5$\mathrm{kW} \mathrm{CO} 2$ laser operating in pulsed mode. Samples were cut into cylindrical sections approximately $120 \mathrm{~mm}$ long and $10 \mathrm{~mm}$ in diameter. Argon gas at 1 bar pressure shielded the melt pool thus avoiding oxidation. The workpiece was rotated with a DC motor fixed to a table moving perpendicular to the laser irradiation direction. Figure 1 schematically represents the laser modification process. The laser beam was kept perpendicular to the workpiece during laser irradiation to maximise the absorbance and ensure uniform conditions for processing [15]. Tangential and linear velocities were kept constant at $270 \mathrm{~mm} / \mathrm{s}$ and $46 \mathrm{~mm} / \mathrm{min}$ respectively, in order to keep the surface spot overlap constant at $0 \%$. A $90-\mu \mathrm{m}$ spot diameter was used for all the experimental runs by focusing the Gaussian beam on the surface of the workpiece. No overlap was used in this study in order to aid the relating 
of beam fluence levels to the corresponding microstructural effects. The pulse repetition frequency was kept constant at $3 \mathrm{kHz}$ so as to control

Table 1: Chemical composition of AISI 316L stainless steel

\begin{tabular}{c|ccccccccccc} 
Element & $\mathbf{C r}$ & $\mathbf{N i}$ & $\mathbf{M o}$ & $\mathbf{M n}$ & $\mathbf{S i}$ & $\mathbf{C o}$ & $\mathbf{N}$ & $\mathbf{S}$ & $\mathbf{P}$ & $\mathbf{C}$ & $\mathbf{F e}$ \\
\cline { 2 - 11 } $\mathrm{Wt} \%$ & 16.82 & 10.14 & 2.03 & 1.51 & 0.55 & 0.08 & 0.061 & 0.03 & 0.03 & 0.02 & Bal.
\end{tabular}

the residence time by varying the duty cycle independently. Residence time, $R$, is the equivalent of exposure time for continuous mode lasers and can be calculated as follows [16]: $\mathrm{R}=\tau \mathrm{D}_{\mathrm{b}} \mathrm{PRF} / \mathrm{V}_{\mathrm{b}}$, where $\tau$ is the pulse width (s), $D_{b}$ is the diameter of the beam spot $(\mathrm{m}), \mathrm{PRF}$ is the pulse repetition frequency $(\mathrm{Hz})$ and $\mathrm{V}_{\mathrm{b}}$ is the scan speed $(\mathrm{m} / \mathrm{s})$.

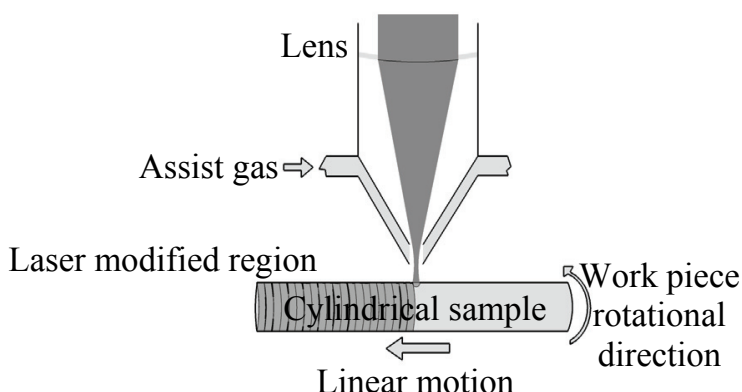

Figure 1: Schematic of the laser processing set-up.

A $2^{5}$ design of experiment was implemented varying the two factors irradiance and residence time. Five levels of each of these factors were used between 7.86 to $23.58 \mathrm{MW} / \mathrm{cm}^{2}$ and 50 to $167 \mu$ s respectively.

The parameters corresponded to an energy fluence of

393 to $3930 \mathrm{~J} / \mathrm{cm}^{2}$ and surface temperature of 1003.8 to $5577 \mathrm{~K}$ according to the surface temperature prediction equation, Equation (1) $[5,17]$ : where $\mathrm{A}$ is the absorption coefficient, $\mathrm{P}$ the laser power $(\mathrm{W}), \mathrm{k}$ the thermal conductivity $(\mathrm{W} / \mathrm{m}-\mathrm{K}), \mathrm{V}_{\mathrm{b}}$ the scan speed $(\mathrm{m} / \mathrm{s}), \mathrm{t}$ the time, $\mathrm{t}_{1}$ the heating time, $\mathrm{t}_{0}$ the time necessary for heat to diffuse over a distance equal to the laser beam radius on the workpiece surface, $t_{0}=R_{b}{ }^{2} / 4 \alpha$, $z$ the coordinate perpendicular to the treated surface and $z_{0}$ is the distance over which heat can diffuse during the laser beam interaction time.

Cross-sectional microscopy views were achieved by sectioning the sample perpendicular to the longitudinal axis. Samples were polished and etched using glyceregia to reveal the grain boundaries for microscopy. Microstructure analysis was carried out using an optical microscope (Reichart Me F2) and scanning electron microscope (SEM), Carl Zeiss, EVO LS15. Surface mean value roughness (Ra) measurements were performed using a stylus profilometer, Civil Instruments TR200, with stylus tip radius of $2 \mu \mathrm{m}$. Sampling and evaluation lengths used were $0.8 \mathrm{~mm}$ and $4 \mathrm{~mm}$ respectively. The roughness was measured according to ISO 4287/4288.

$$
T(z, t)=T_{0}+\frac{A P}{2 k \pi V_{b} \sqrt{t\left(t_{t}+t_{0}\right)}}\left[\exp \left\{-\frac{\left(z+z_{0}\right)^{2}}{4 \alpha t}\right\}+\exp \left\{-\frac{\left(z-z_{0}\right)^{2}}{4 \alpha t}\right\}\right] \operatorname{erfc}\left(\frac{z+z_{0}}{\sqrt{4 \alpha t}}\right)
$$
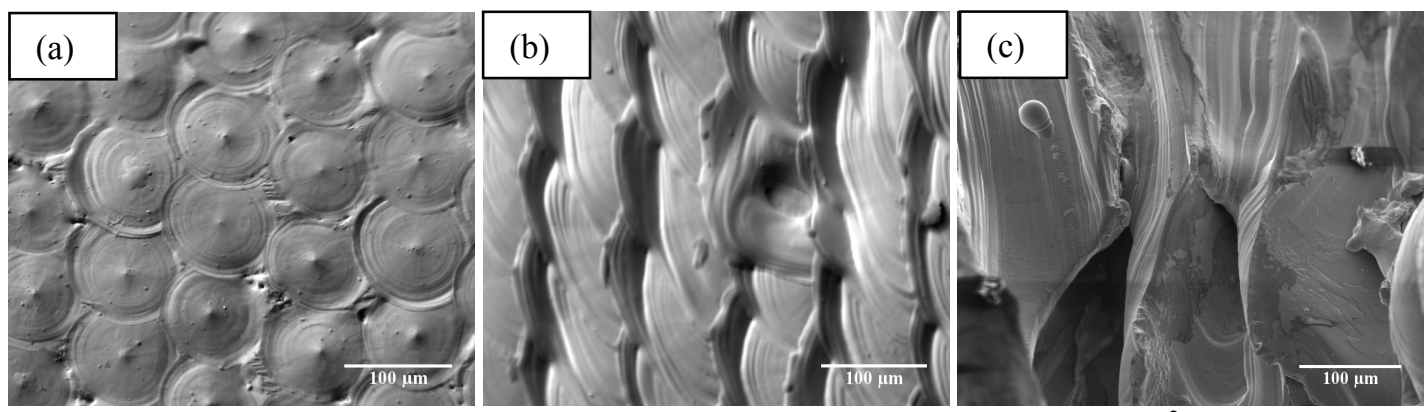

Figure 2: Effects of energy fluence on surface melting (a) $524 \mathrm{~J} / \mathrm{cm}^{2}$,

(b) $1048 \mathrm{~J} / \mathrm{cm}^{2}$ and (c) $2096 \mathrm{~J} / \mathrm{cm}^{2}$. 

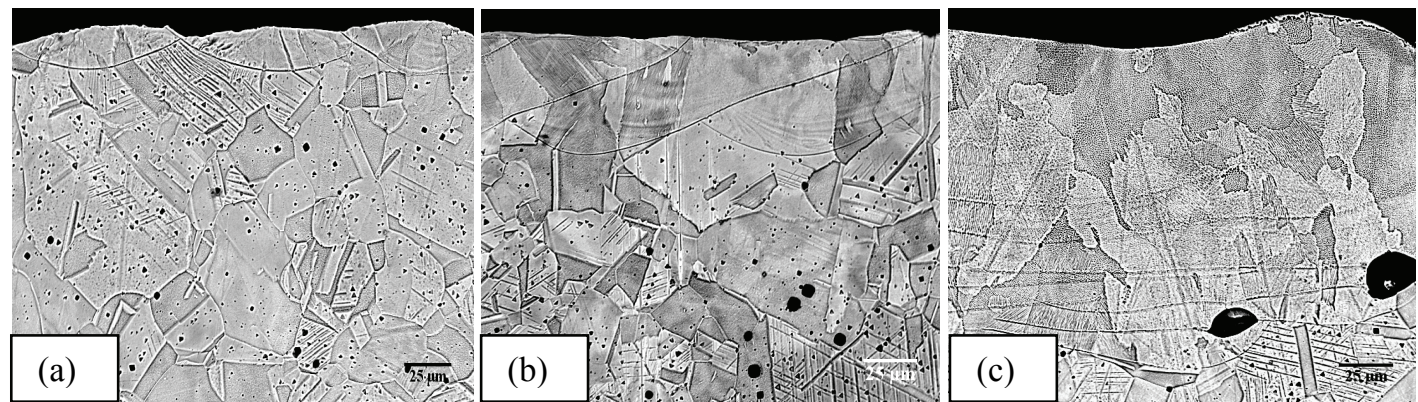

Figure 3: Back scatter SEM images of transverse cross-sectional microstructure corresponding to processed surface shown in Figure 2 (a), (b) and (c).

\section{RESULTS AND DISCUSSION}

\subsection{Microstructure / Thermal Distribution}

Surface structure and cross-sectional microstructure of the laser processed steel at 524,1048 and $2096 \mathrm{~J} / \mathrm{cm}^{2}$ are displayed in Figures 2 and 3 respectively. Figure 2 illustrates the surface melting induced by the laser treatment at increasing energy fluence. The surface temperature directly under the surface was estimated, using Equation 1, to be approximately 1190, 2001 and $3111 \mathrm{~K}$ for figures 2 and 3 (a) (b) (c) respectively. Figures 2 and 3(a) show laser marking features with localized melting effects and minimal depth of processing. No grain structure changes are visible in figure 2 (a) with only heat affected zones visible without any real evidence of melting of the surface. At $\sim 2000 \mathrm{~K}$, figures 2 and 3 (b) illustrate homogenised melting with increased depth of processing and overlapping becoming visible. Sample rotation parameters were set to produce $0 \%$ overlapping of the melt pool as represented in figures 2 and 3 (a). The overlapping effects shown in figure 2 and 3 (b) represent melt pools wider than the original spot size being produced due to elevated irradiance and thermal properties of the material. Grain direction orientation is also visible at $1048 \mathrm{~J} / \mathrm{cm}^{2}$ although grain size is not significantly changed due to high quenching rates. Figures 2 and 3 (c) depict the surface and cross-section microstructure at an energy fluence approximately 4 times larger than (a) with surface temperature approximately $3111 \mathrm{~K}$. An increased depth of processing is observed, ablation is also visible in Figure 2 (c) due to increased irradiance values. Grain size and orientation is significantly altered due to slow cooling rates and higher residence times. The overlapping boundaries visible at low energy fluence can be seen in Figure 3 (c) as layers representing different isotherms.

\subsection{Melt pool size analysis}

Figure 4 highlights the effects of residence time on the depth of processing at varying irradiance values. Augmentation in melt pool depth is fairly linear with increasing residence time for all irradiance values. High residence times result in increased interaction of laser beam and surface of the material thus increasing the cooling rate and melting region.

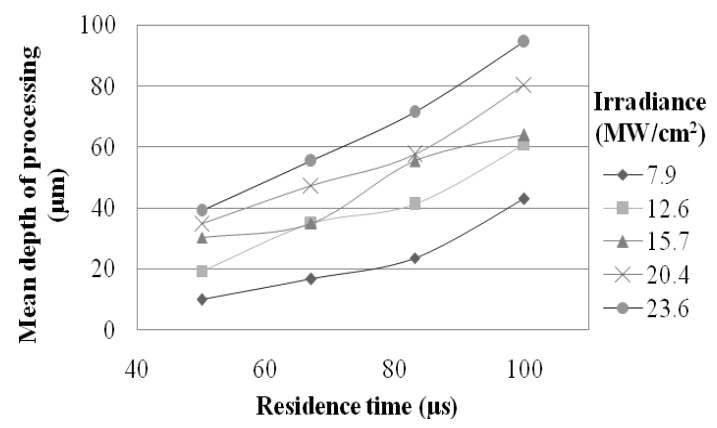

Figure 4: Effects of Irradiance and residence time on melt pool depth

To clearly illustrate the effects of irradiance and residence time on the depth of processing and roughness, the same energy fluence of $1310 \mathrm{~J} / \mathrm{cm}^{2}$ with two different combinations of irradiance and residence time produced the micrographs shown in Figure 5. A combination of low peak power $(0.5 \mathrm{~kW})$ and high residence time $(167 \mu \mathrm{s})$ produced a melt pool depth of $115 \mu \mathrm{m}$, see Figure 5 (a). The depth was approximately double that of a combination of high peak power $(1 \mathrm{~kW})$ and low residence time $(83 \mu \mathrm{s})$ which produces a depth of $57 \mu \mathrm{m}$, see Figure 5 (b). The results show that residence time has a greater effect on depth of processing compared to irradiance. In order to improve the tribological properties, very high cooling rates are required. The cooling rates 
can only obtained at very low exposure time with extremely high irradiance. Using the minimum permissible limit of $27 \mu$ s residence time, an average power of $1000 \mathrm{~W}$ would be required, which is over the limit of the laser unit. A way to increase the irradiance would be by improving the absorbance of stainless steel as only $3.5 \%$ absorbance was estimated using the surface temperature prediction in Equation 1 and known literature [15].
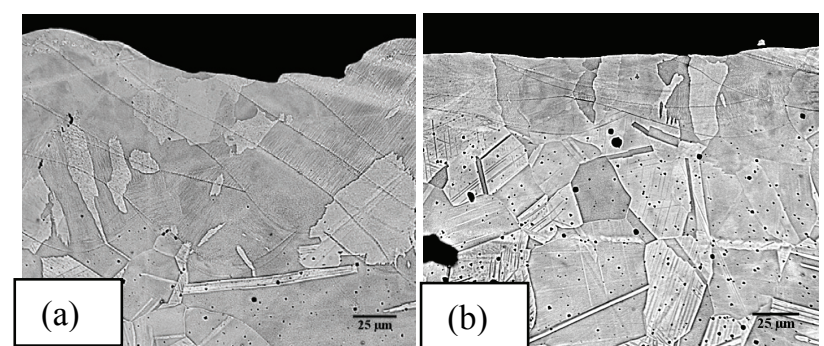

Figure 5: BSE cross-section micrographs; energy fluence $\left(1310 \mathrm{~J} / \mathrm{cm}^{2}\right)$ with (a) peak power $(0.5 \mathrm{~kW})$ and residence time $(167 \mu \mathrm{s})$ and $(\mathrm{b})$ peak power $(1 \mathrm{~kW})$ and residence time $(83 \mu \mathrm{s})$

\subsection{Roughness Analysis}

The average roughness of untreated stainless steel was estimated to be $1.96 \pm 0.26 \mu \mathrm{m}$. Figure 6 (a) illustrates the roughness results obtained at varying residence times. The roughness increases as the irradiance is raised for all given residence times. Irradiance is the power density per unit area; therefore higher powers induce ablation which is visibly clear in the microstructure image shown in Figure 2 (c).

At low irradiance $\left(7.9 \mathrm{MW} / \mathrm{cm}^{2}\right)$, varying residence time does not have a major effect on roughness except for the highest $167 \mu \mathrm{s}$. There is no interaction at low residence time due to no melting occurring at low exposure times. Low exposure times have roughness values comparable to the untreated steel since there no melting occurring at these points. The roughness values obtained in this study give a good indication of the melting status of the parameters used in the experiment.
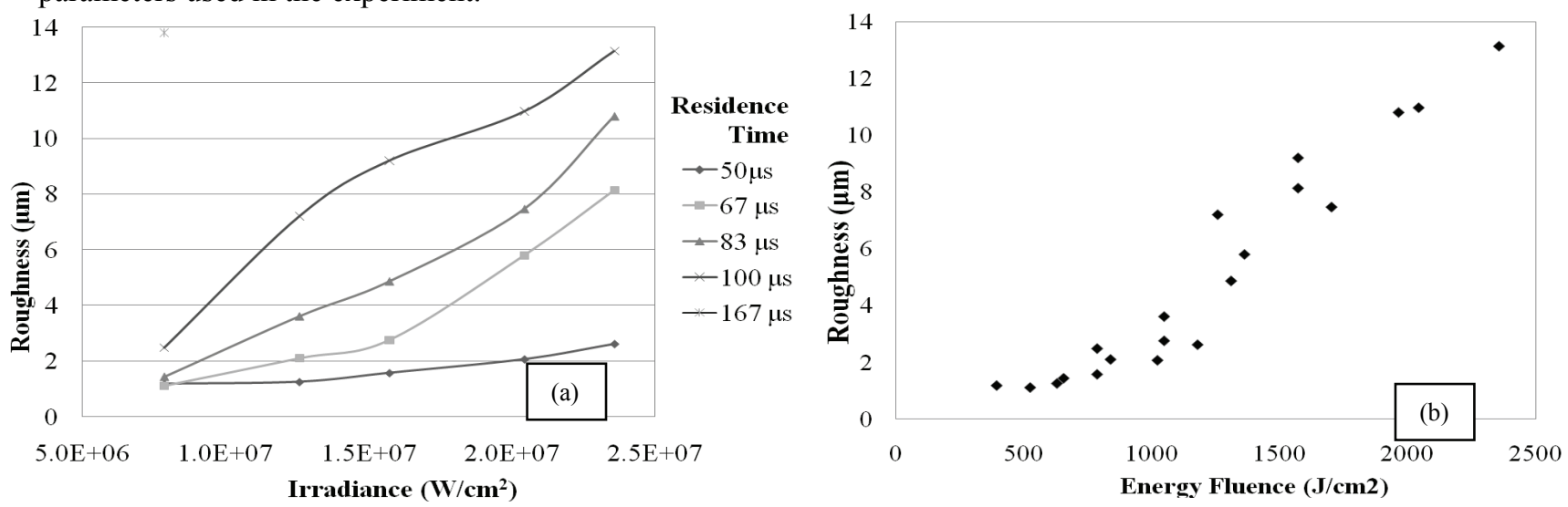

Figure 6: Relationships between average surface roughness and

(a) Irradiance at varying residence times and (b) Energy fluence

Increasing the laser beam exposure time resulted in elevated temperatures on the surface with reduced cooling rates. The characteristics of such a treatment is increased roughness due ablation occurring on the surface. The highest residence time implemented in the experiment, $167 \mu$ s, resulted in extremely high roughness outputs. The equipment used for the roughness measurement was unable to detect the resulting mean values of the sample treated at irradiance values over $7.9 \mathrm{MW} / \mathrm{cm}^{2}$. A strong correlation is also visible between roughness and energy fluence used in the experiment regardless to the exposure time; see Figure 6(b). The first three points on the graph represent regions not melting due to the low energy fluence. A linear increase in roughness is observed with higher energy fluence results showing a strong correlation.

\section{CONCLUSION}

A low energy fluence (less than $629 \mathrm{~J} / \mathrm{cm}^{2}$ ) had no melting effect on the surface of the stainless steel regardless of the irradiance and residence times used. Low depth of processing was observed with no physical change in grain structure detected. Mid level energy fluence of $1048 \mathrm{~J} / \mathrm{cm}^{2}$ produced uniform melting on the surface. Melt pool depth reached approximately $60 \mu \mathrm{m}$ with visible overlapping induced by increased melting areas larger than the 
laser spot size. High levels of energy fluence, exceeding $2096 \mathrm{~J} / \mathrm{cm}^{2}$, produced changes in grain size and orientation and higher depth of processing (up to $130 \mu \mathrm{m}$ ). Larger grains were also observed in the processed regions and evidence of ablation was visible on the surface. Roughness and melt pool depth increased with higher levels of both irradiance and residence time.

From the study it is clear that a combination of low levels of irradiance and high residence times were best for laser processing biomedical implant structures. The resultant structure is characterised by lower roughness and moderate depth of processing. The main focus of future studies would be achieving an improved surface with improved tribological properties and then using the process mapping detailed in this investigation to obtain optimum surface roughness and depth of processing parameters.

\section{REFERENCES}

[1] L. Hao: Laser surface treatment of bio-implant materials (Wiley 2005)

[2] P.A. Dearnley: Proceedings of the Inst. of Mech. Eng. -- Part H, Vol.213, 107-135 (1999)

[3] X. Liu, P.K. Chu, C. Ding: Materials Science and Engineering: Vol.47, 49-121 (2004)

[4] L. Hao, J. Lawrence: Journal of Biomedical Materials Research - Part B Vol.73, 148 (2005)

[5] J.F. Ready, D. Farson: Handbook of laser materials processing (LIA 2001)

[6] W.M. Steen, K.G Watkins: Journal De Physique IV, Vol.3, 581 (1993)

[7] J.D. Majumdar: Surface and Coatings Technology, Vol.179, 297-305 (2004)

[8] J.D. Majumdar, I. Manna: Laser processing of materials, Sadhana, Vol.28, 495 (2003)

[9] S.N. Aqida, D. Brabazon, and S. Naher: Proceedings of Advances in Materials and Processing Technologies (2009)

[10] N. Parvathavarthini: J. Mater. Eng. and Performance, Vol.10 (1), 5-13 (2001)

[11] X.L. Wu, Y.S. Hong: Metal. Mater. Trans. A 31, 3123-3127 (2000)

[12] M. M. Shalabi: Journal of Dental Research Vol. 85, 496-500 (2006)

[13] H. J. Ronold.: Biomaterials Vol. 24, pp. 4559-4564 (2003).

[14] D. Murray, T. Rae, N. Rushton: J. Bone Joint Surg. Br. Vol 71, 632-637 (1989)

[15] T. Mahank: Laser glazing of metals and metallic and ceramic coatings (2004)

[16] A.N. Samant: J.Material Processing Technology. Vol.209, pp. 5060-5067 (2009)

[17] J.S. Selvan, G. Soundararajan, K. Subramanian Surface and Coatings Technology, Vol. 124, pp. 117-12 $7(2000)$ 